trending up; however, without attaining statistical significance $(\mathrm{p}=0.45)$.

Conclusion Our nationally represented sample showed no difference in outcomes in medically, and EVT treated groups among gender despite prior literature suggesting poor outcomes in the EVT group. Patient selection for EVT should be individualized based on patient presentation. Our analysis showed CVT treated with EVT hospitalization is trending up in the US.

Disclosures S. Patel: None. N. Desai: None. M. Pervez: None.

\section{E-005 NORMALIZATION OF ELEVATED IDIOPATHIC INTRACRANIAL VENOUS PRESSURES AFTER MANOMETRY AND HIGH-VOLUME LUMBAR PUNCTURE IN A PATIENT WITH PSEUDOTUMOR CEREBRI}

${ }^{1} \mathrm{M}$ Brown*, ${ }^{2} \mathrm{~T}$ Wolfe. ${ }^{1}$ Radiology, Aurora St. Luke's Medical Center, Milwaukee, WI; ${ }^{2}$ Neuro-Interventional Radiology, Aurora St. Luke's Medical Center, Milwaukee, WI

10.1136/neurintsurg-2020-SNIS.41

Treatment of idiopathic intracranial hypertension (IIH), also known as pseudotumor cerebri, generally prioritizes alleviating headaches and preserving vision. If medical management is unsuccessful, possible surgical treatment options depending on symptom severity include serial lumbar punctures, venous sinus stenting, optic nerve sheath fenestration (ONSF), and cerebrospinal fluid (CSF) shunting. Venous sinus stenting has remained controversial as cerebral vein stenosis may not be a primary cause.

In our case, we present a 38-year-old female with known pseudotumor cerebri who underwent a diagnostic cerebral angiogram with manometry and high-volume lumbar puncture. Pre-lumbar puncture manometry demonstrated venous pressures throughout the left and right transverse sinuses and distal superior sagittal sinus ranging from $25-30 \mathrm{mmHg}$ with associated bilateral transverse-sigmoid junction stenoses. The patient was then placed in the lateral decubitus position and a lumber puncture at L2-3 was performed. Opening pressure was measured at $29 \mathrm{~cm} \mathrm{H} \mathrm{H}_{2} \mathrm{O}, 30 \mathrm{~mL}$ of clear CSF was removed, and closing pressure was measured at $8.5 \mathrm{~cm} \mathrm{H}_{2} \mathrm{O}$. Post-lumbar puncture manometry demonstrated normalization of respective venous pressures ranging from $8-12 \mathrm{mmHg}$ with resolution of associated bilateral transverse-sigmoid junction stenoses and improved venous sinus calibers. Following the procedure, the patient reported resolution of headache.

These results indicate that cerebral venous stenoses in the setting of IIH may be a secondary phenomenon. Thus, patients may benefit from CSF shunting as a primary surgical treatment option. In order to establish treatment efficacy, future studies could evaluate for stenosis and pressure gradient recurrence after CSF shunting.

Disclosures M. Brown: None. T. Wolfe: None.

\section{E-006 TUNING OF INNOVATIVE IN-VITRO MODEL MATERIALS TO MIMIC TISSUE PROPERTIES}

${ }^{1} \mathrm{~N}$ Norris ${ }^{*},{ }^{2} \mathrm{Smith},{ }^{2} \mathrm{C}$ Settanni, ${ }^{2} \mathrm{~W}$ Merritt, ${ }^{1,2} \mathrm{~T}$ Becker. ${ }^{1}$ Aneuvas Technologies Inc., Flagstaff, $A Z_{;}{ }^{2}$ Northern Arizona University, Flagstaff, $A Z$

10.1136/neurintsurg-2020-SNIS.42

Introduction/Purpose In-vitro models help test new medical devices for use in numerous cardiovascular and neurovascular

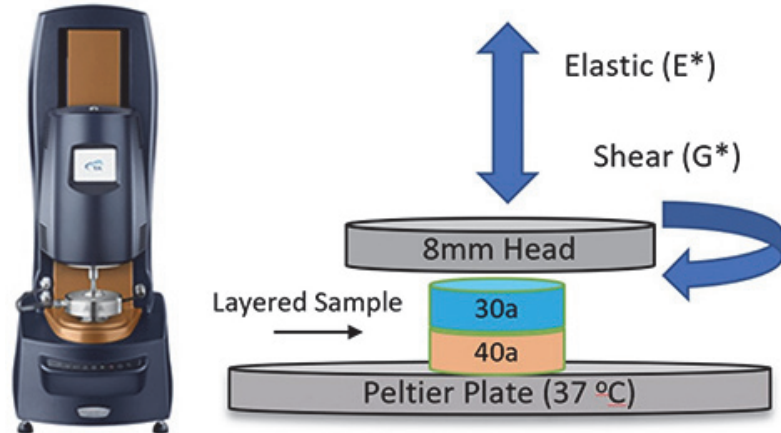

Abstract E-006 Figure 1 Left - Hybrid Rheometer. Right Rheometer Setup for Layered Sample

treatments. These models are also commonly used for the training of surgeons on innovate devices and new surgical procedures. These current vessel-training models cast from human vasculature with anatomical accuracy; however the materials often used for casting (i.e. silicone and glass) do not accurately simulate mechanical properties of tissue such as: vascular compliance (modulus), tensile and compressive strength, wall friction (lubricity), and hardness seen in native human vasculature. Thus, a more tunable and comprehensive in-vitro model material is needed to better understand how endovascular devices (i.e. microcatheters/wires, thrombectomy devices, coils, stents, flow diverters, and liquid embolics) interact with the vessel wall during delivery. An innovative 3D printed acrylic co-polymer (VeroClear ${ }^{\circledR}$ Agilus $30^{\circledR}-$ VCA30) can be tuned to match the mechanical properties of human vessels.

Materials and Methods Differing hardnesses of VC-A30 are layered and mechanically characterized with a hybrid rheometer (DHR 2, TA Instruments). The results are compared to replicated test results of donated 'fresh' human cadaveric tissue samples (Common Carotid Artery). Via the rheometer, data is collected for luminal wall friction, radial compliance, shear modulus, and elastic modulus (figure 1). Properties of cadaveric vessels and model materials are statistically compared to the VC-A30. The biomaterial layering is altered by varying the thickness of the VC-A30 layer thicknesses (biomaterial tuning) to mimic the mechanical properties of the cadaveric vasculature within statistical equivalence. Once successfully optimized, the biomaterials are manufactured into flow models and will additionally be validated by partnered neurointerventionalists to ensure that the model has realistic catheter trackability and is anatomically accurate.

Results The VC-A30 materials in the previous studies simulated the compliance and mechanical properties of human vasculature more closely than existing in-vitro silicone materials. VC-A30 is 3D printed to achieve accurate anatomical features; moreover, this $3 \mathrm{D}$ printed material is layered to simulate the lubricious inner lumen of a vessel, with varying hardness profiles to mimic vessel compliance and strength. This assembled structure simulates the layers of human vasculature and its variable properties.

Conclusion The utilization of novel biomimetic materials within this in-vitro vascular flow model will allow for more relevant benchtop testing of endovascular devices. These models have the potential to generate more accurate data on device performance and may reduce the need for costly in-vivo studies. 